\title{
Usability Considerations in Developing a Graphic Interface for Intra Office Communications
}

\author{
Pradeep Yammiyavar and Piyush Jain \\ Indian Institute of Technology Guwahati, Guwahati - 781039, India \\ \{p.jain, pradeep\} aiitg. ernet. in
}

\begin{abstract}
This paper outlines the basis of incorporating functional features in a new GUI based software under development for addressing comprehensive communication and interaction needs within an office environment. Bench marking of features in existing communication software products such as Microsoft Outlook, IBM Lotusnotes, Office Communicator, Mozilla Thunderbird etc. was done by asking a set of questions related to the usage of these existing softwares. Usability issues were identified through a user survey involving 30 subjects of varied profiles (domain, designation, age etc.) in a corporate office. It is posited that existing software products that have been developed for a universal market may be highly underutilized or have redundant features especially for use as an intra office (within the same office) communication medium. Simultaneously they may not cater to some very contextual requirements of intra office communications. Based on the findings of the survey of feature preferences \& usability of existing products, a simple 'person to person' communicating medium for intra office situations was visualized with a new interactive GUI. Usability issues that need to be considered for a new intra-office product have been brought out.
\end{abstract}

Keywords: Intra Office Communications, Usability, Interface Design, Requirement Analysis.

\section{Introduction}

Effective communication in between its employees contributes to proper functioning of the organization. One can term this as Intra Office Communication. Technically, communication is defined as the process of transfer of information between the communicating entities. Much of the work inefficiency in offices can be traced to poor and hurried communication leading to incomplete or misunderstanding.

There are a plethora of communication services on the net that cater to communication needs in offices and organizations. Email, List servers, Newsgroups, Web Conferencing, Internet Relay Chat (IRC, also termed as Instant Messaging (IM)), Internet Phone (Internet Protocol (IP) Calling), Internet Radio, Desktop Video Conferencing and File Transfer Protocol (FTP Client) are few examples of the large number of 'Web-Based Electronic Collaborative Tools'. After the advent of the Web 2.0, newer concepts of social interaction such as Social Networking (Face book, LinkedIn etc.), Blogging (Wordpress, Blogspot etc.), Micro Blogging (Twitter), RSS Feeds etc. have 
evolved [3]. Many of these are jostling for attention as personal cum semi-official communication enabling products. This has turned out in making communication a complex and an incoherent process. The basic dissimilarities at the structural and interfacial levels aggravate the problem further. There are learnability and usability issues which remain largely unaddressed in the new software. All this adds to the confusion for a user who wants a simple intra-office product to communicate between co employees.

It was hypothesized that many of the existing features of available software might be classified as redundant, repetitive or unnecessary in terms of usability.

\section{Identifying Typical Intra Office Communication Needs}

The need to identify typical intra office communication requirements come from the extensively diverse ways of communication adopted in an office setting consciously as well as unconsciously. A threefold research strategy was adopted to accomplish this task. This involved a study of the existing products, a rigorous user study and finally prioritizing the features of a hypothetical new product. This would be then followed by conceptualizing the GUI for a new office utility.

\subsection{Software Utility Analysis}

A large number of serving software applications were found to be used by office workers. A list of 10 most popular office soft wares and web services were short listed. Heuristic evaluation targeting the features of each product was done. The applications short listed were Microsoft Outlook, IBM Lotus Notes, Mozilla Thunderbird, Office Communicator, Google Talk, SSH Client, Message Pal, Gmail (Web), LinkedIn (Web) and Facebook (Web). Some of the important and notable features and characteristics of the present products are as listed below:

Emailing: Email is the most common and preferred collaborative communication tool in use today. Besides its basic functionality of being an asynchronous communication tool [1], it is also being used increasingly for information systems' management, coordination and collaboration tasks in organizations (Ducheneuaut \& Bellotti 2001). Email is a crucial tool because virtually everybody who has ever touched a computer knows about email besides it is relatively easy to use. The interesting observation, however, is that most of the added utilities to email programs like task managing, calendar, email flagging, email tracking, temporal information organization, time management etc. are found to be redundant as compared to its basic functionality viz. manipulate individual messages and message threading. Compatibility issues of the email service with other software utilities are partially responsible for this (Gwizdka 2004).

Instant Messaging (IM): A form of real time (synchronous) communication [2], instant messaging evolved as posterity to emailing. Communication is possible between two or more users and with one or many users simultaneously. Newer Instant Messengers also come up with features like file transferring, voice messaging, IP calling, video chatting and custom 'status message' settings. Instant 
messaging is preferred more for informal and quick conversations. IM language is one of the more noted features of instant messaging which involves extensively the use of evolved abbreviated forms, smiley faces and symbols (like*(star) for spelling correction) for better interaction.

Social Networking: This new wave of internet based social interactions catalyzed particularly after the advent of Web 2.0. In the past five years, it has rocketed from a niche activity into a phenomenon that engages tens of millions of internet users socially connected to each other[4][5]. While networks like Facebook and Orkut are already popular for creating social groups, mutated versions of the same have evolved for office environments and professional work too in the form of services like LinkedIn. Structurally, a social network is made of nodes interdependent on each other on one or more parameters. The most unique feature of social networking is the concept of profile management on a personal and community level. With other add-ons like community forums, photo sharing, games, personal recommendations, social networking has revolutionized the world of internet based interactions.

File Sharing: File sharing facility is actually dependent upon the type of network shared between the users (LAN, internet etc.) and the size of the file transferred. While typical email services provide file transfer facilities of about $10-15 \mathrm{Mb}$ space per email attachments, file transfer protocol (FTP) clients are standalone services specifically designed for file sharing and hosting. On an intra office level, FTP clients contextual to local intranet as often used to share official work related material. This is often practiced as a team activity rather than just person to person communication.

Voice over Internet Protocol (VoIP): More frequently termed as IP calling, voice over internet protocol is one of the newer features found in modern internet based interaction services. It provides a unique telecommunication capability by exceeding the standards of traditional telephony. IP Phone is a technology in itself. Over half of the fortune 500 companies are deploying VoIP phones and over 10,000 organizations have deployed the technology with millions of IP phones acquired. VoIP is becoming a mainstream technology in the world of commerce and is likely to spill over beyond corporations. The most advantageous features of VoIP are the reduced operational expenses and their unified messaging capabilities.

Video Conferencing (Vcon): Vcon is a step next to VoIP which involves interactive telecommunications between two or more remote locations with visuals. However with some serious usability concerns like privacy and reciprocity, audio-video quality, audio localization gaze awareness and eye contact etc. Vcon has yet to take off on a big scale.

RSS Feeds (Web Feeds): Really Simple Syndication Feed or Web Feeds is something that found popularity during the time 2005-06 and gained sudden momentum with blogging. RSS feeds are particularly relevant to offices and organizations due to their capability of rapid news capture and propagation. However, RSS feeds have often been found to score very low on usability standards, especially with the process of subscribing and unsubscribing them. Most office users are simply ignorant what RSS feeds are in spite of encountering the term and the popular logo time and again on the internet. 


\subsection{User Study}

In the previous section we gave a brief overview of a few utility features of the existing systems. To understand the attitudes and issues of users using the above products, a user survey was conducted. We sampled a set of 30 different users of working in an internationally known Indian Information Technology company in Benguluru. Data gathering sessions composed of questionnaires and contextual inquiries through interviews. After an initial pilot test of the questionnaire amongst 5 selected users, the questionnaire was refined and launched online to gather larger volumes of quantitative data. The questions aimed at finding out the most used and most preferred features amongst different competitive software. Data about their working experience and expertise with different software was also collected. Users were differentiated on the basis of their age groups, work experience, gender, designation and work type.

We conducted contextual inquiry sessions with the selected users for about 30 minutes each, which included responding to a printed questionnaire. Occasionally, users were also asked to perform a specific set of tasks on some of the previously mentioned software.. Users often faced difficulty in handling software they had not worked upon previously. Experienced users were able to provide valuable insights.

Following are reproductions of selected questions from the study and replies elicited during the contextual inquiry sessions:

1. What are the different ways (physical/electronic/web based) in which you communicate with people in your office?

There were different responses. A typical response from a user for the above question: "E-mail is the most essential primary tool for communication.

Intranet messenger is used for informal communication", "E-mail, IM, SMS

Phone", "E-mail, Phone, FAX, Video Conferencing......."

2. What all software are you acquainted with as communicating software? Please mention the time (in months) you have been using each of these.

The responses are compiled in table 1.

Table 1. Software usage pattern over time of acquaintance (in months)

\begin{tabular}{ccccccc}
\hline & $\begin{array}{c}\text { MS } \\
\text { Outlook }\end{array}$ & $\begin{array}{c}\text { Office } \\
\text { Communicator }\end{array}$ & Gtalk & $\begin{array}{c}\text { Mozilla } \\
\text { Thunderbird }\end{array}$ & $\begin{array}{c}\text { IBM } \\
\text { Lotusnotes }\end{array}$ & Y!Messenger \\
\hline $\begin{array}{c}\text { Number } \\
\text { of Users }\end{array}$ & 30 & 21 & 18 & 12 & 10 & 8 \\
$\begin{array}{c}\text { Average } \\
\text { Time } \\
\text { since }\end{array}$ & 10.0 & 8.5 & 13.0 & 8.0 & 8.5 & 12.0 \\
using & & & & & & \\
\hline
\end{tabular}

3. Which is your preferred way (software/utility) of communicating to your office mates amongst those listed above? Please rank in order of preference.

For a particular user we awarded 10 points for rank 1, 8 for rank 2 etc. Total points scored by the software averaged over the number of users who voted, gave the average points scored by each software. Average rank is the order of average points scored, 1 being the most preferred. 
Table 2. Preferential list of Software. Ranks averaged over user preferences.

\begin{tabular}{ccccccc}
\hline & $\begin{array}{c}\text { MS } \\
\text { Outlook }\end{array}$ & $\begin{array}{c}\text { Office } \\
\text { Communicator }\end{array}$ & Gtalk & $\begin{array}{c}\text { Mozilla } \\
\text { Thunderbird }\end{array}$ & $\begin{array}{c}\text { IBM } \\
\text { Lotusnotes }\end{array}$ & Y!Messenger \\
\hline $\begin{array}{c}\text { Average } \\
\text { rank }\end{array}$ & 1 & 2 & 3 & 4 & 5 & 6 \\
\hline
\end{tabular}

4. Do you depend on some specific modes (like email for example) to communicate to certain people in your office? Or you get along with whatever is the easiest or the quickest?

Typical responses were as follows: "Formal messages, without any choice have to be corresponded as e-mails. I prefer IM for general communication", "Of course! Like you have to use an FTP client for transferring large files or working on shared folders", "I hesitate using IM to communicate with my project manager, or other senior officials", "I really don't like typing mails or even IMs, I prefer the office phone at most times".

5. How do you rate Microsoft Outlook? Any particular problems that you have faced while using it? Any suggestions about the same?

Typical responses: "I am not aware of many features like Journal, RSS, Labels etc. I am really not keen to explore much of these", "Outlook 2003 does not support previewing attachments and that's really annoying”, "I don't know how to make a signature, but I've seen mails bearing the same”, "Search is not powerful enough, like these days we have searches that show suggestions instantly for every word. Then, I can't search IDs for typing names"

6. Would you prefer having one integrated system (if it were to exist) for total communication requirements? Or are you comfortable and happy with the state of art facilities?

\section{Results of preference for an Integrated Comprehensive System}

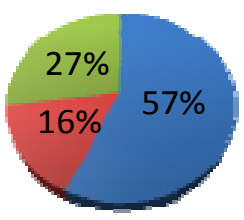

Yes

No

Don't Know

Fig. 1. User response to the proposal for a new comprehensive system which address their complete communication needs in an office environment 
7. Following is a set of features that we proposed to incorporate in designing an integrated futuristic communication application. On a scale of 1 to 10 , with 10 indicating highest degree of preference, rate the features.

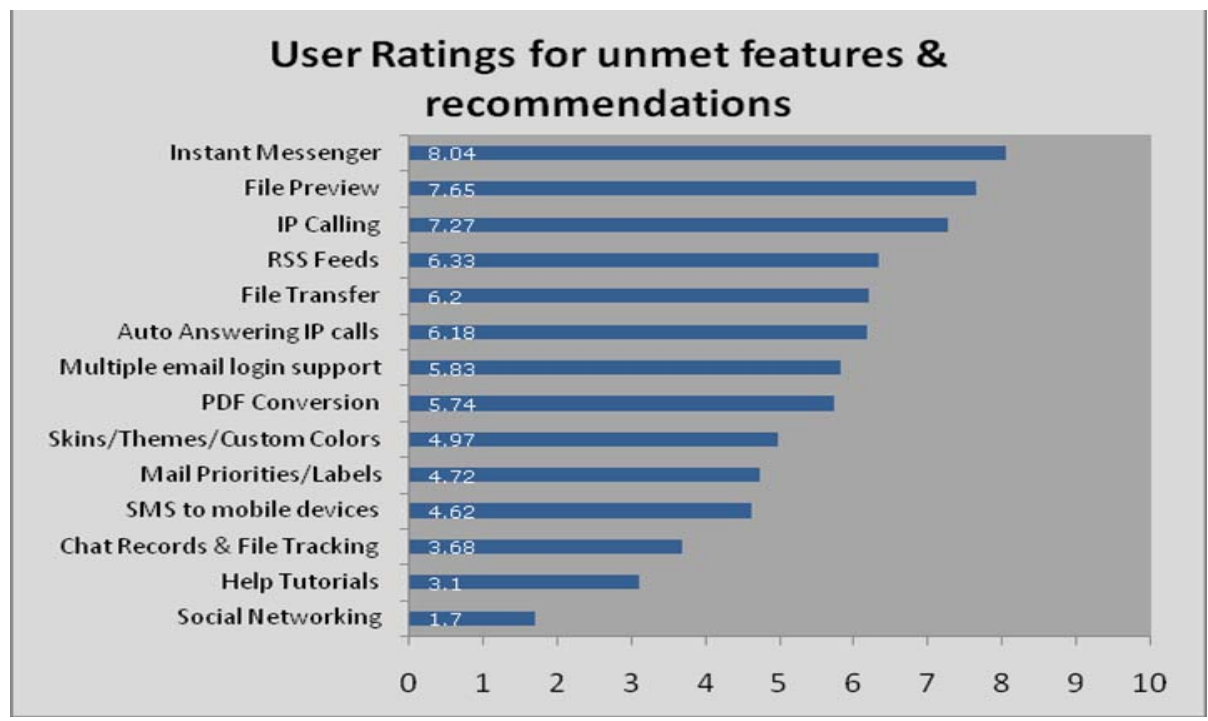

Fig. 2. Average users' ratings for features in office communication software, ('Outlook')

Contextual inquiries often resulted in open ended qualitative questions and discussions when the user would precisely point on the screen, the feature he/she failed to understand or the problem faced. This provided valuable inputs to understand the lower levels of interactivity existing between the user and the software.

\section{Results of the User Study}

The results of the User Study conducted in numerous contextual inquiry sessions, online questionnaires and heuristic evaluations of varioussoftware were interpreted on both qualitative and quantitative levels. An Attribute List of the recommended features for a new office communication system was then prepared. It also threw light on the unmet needs.

The primary communication needs identified (in decreasing order of priority) were as follows: (1) Emailing (2) Instant Messaging (3) Phone Calls (4) SMS (5) FAX (6) Video Conferencing. The most common software products in use (decreasing order of usage) were Microsoft Outlook, Microsoft Office Communicator, GTalk Client, Mozilla Thunderbird, IBM Lotus Notes and Yahoo Messenger.

It was observed that Instant Messaging topped the list of unmet needs and users often had to resort to separate software like Skype and Google Talk to serve this purpose. More interestingly, unlike other features, instant messaging was backed by almost all types of personas be it consultants, managers, coders (developers) or designers etc. We now have a look at some of our significant qualitative observation: 
A majority of users preferred using a single application that could cater to complete communication needs rather than operate multiple software for different communication requirements. Although the above ratings show the priority of features on a cumulative scale, users differentiated in their preferences on a individual level. For example someone like a manager rated News Feeds quite higher than a software engineer. Preferences varied significantly over users with different age groups. While younger people rated rather non functional features like skins/custom colors higher (perhaps to keep the ambience more lively and enjoyable), experienced people almost discarded it. Tailorability (Customizability) was one important unmet need noticed which remained unaddressed by any software so far.

Social Networking, initially speculated to be one of the major trends of contemporary communication systems was rejected by all types of users. When probed for the reason they accounted for the unnecessary expenses of time and over indulgence in social network activities. People did want their web based social networks to remain intact in place at the same time disapproved introducing an intra office social network system. They wanted to keep the social network needs different from office work needs.

Microsoft Outlook was although the most prevalent software system used amongst the surveyed office goers but this was only because of that particular company's policy to use it in their offices and not because of the users' choice. New recruits especially faced this problem time and again and had to consult their co workers to help them out in different situations.

\subsection{Inferences and the Attribution List for a New GUI}

A number of interesting inferences could be drawn from the above survey results. We discovered that what users require is an application affording all-round - '360 degree communication and interaction within the office' without having to go out of their current working GUI screens. However, this does not imply to make a potpourri with all possible flavors of software added to it. Rather we need to select the essential ones which the users actually want. Unnecessary repetition of redundant features offers no advantage. Adding to this, many crucial needs remain unaddressed and lead to the diversification of software.

We discussed in section 2.2.1 how preferences varied considerably with changing user profiles. It can be stated that a section of the users might be quite satisfied with the current state of art viz. the diverse ways of communications and their diverse utilities. There might be another large section of the users who are looking out for a radical change. Same could be posited for the various features. Age, domain and experience play a central role in dictating a person's preferences as reflected in our user testing.

\subsubsection{Tailorability: A Concept That Facilitates the Users Configure for Themselves}

One proposal could be to let the user define his/herown requirements and configure hisown system. We label this feature as Tailorability of the system. Software applications in recent times have been developed to be more flexible. Products like Eclipse and Mozilla Firefox are examples of highly flexible and customizable systems. The 
user has the liberty to adapt the system according to his needs, work practices and environment.

We propose to incorporate tailorability in the new GUI. Flexibility could be built in in the sense that of the several features available only the basic or the most essential ones could be configurable upfront, while the others provided for subjected to user's discretion. This can be achieved by introducing a user interface like a customizable DockBar. Different features like News Feeds, Journal, Remainders, Sticky Notes (Tasks) etc. can be docked on the DockBar as Docklets similar to simple widgets. This not only satisfies the user by giving him the freedom to tailor his/her own system, but also helps in reducing the general clumsiness of the interface and increase usability.

Managing contacts in general was one of the most used and most 'complained about' feature while using a communication system like Outlook. While trying to figure out why this happened, we observed a very basic problem. Every time when a user has to contact a person, he has to first make a choice about selecting the way of communication he needs to use to contact the person. However, communication is a user centered and not a task centered process.

This means that the interface should be so that the user does not have to bother first about selecting one out of the $n$ ways of communication to connect to his contact, but selecting the contact itself. The same should be taken care of when the user wants to contact more than one simultaneously. This is something that can be addressed purely at an interaction design level of the User Interface. Table 3 depicts a list of features and requirements elicited from our study so far. This includes both the present and the proposed features listed in priority of their usage and requirement. Common file formats like .doc, .xls, .pdf, .jpeg etc. are recommended to be preview able prior to downloading them as attachments or repositories. This makes it easier to organize both mails and desktops. Other features include facilitating a single client to manage more than one mail address. Utility tools include features like inbuilt PDF converter, Sticky Notes (Tasks), Auto Answering Feature, Utility widgets like Live Stock Exchange News etc.

Table 3. List of essential features arranged in order of priority

\begin{tabular}{|ll|}
\hline 1. & Emailing \\
\hline 2. & Instant Messaging \\
\hline 3. & IP calling \\
\hline 4. & RSS Feeds \\
\hline 5. & File Sharing \& Previewing \\
\hline 6. & Calendar \\
\hline 7. & Multiple mail login support \\
\hline 8. & Utility tools \\
\hline
\end{tabular}

\section{Conceptualizing a New Intra Office Communication Utility Product}

Based upon our discussion and conclusions in the previous sections, we conceptualized wire-frames of GUIs for a new Intra Office Communication Utility and called it 
Office 360. We started with making different iterations by paper prototyping for a new proposed communication system counting around 10 in number. We then tested each prototype on the parameters of our findings and conclusions. In this iterative process we also made an Information Architecture for the new system. Based on the information architecture we made the various screens and sections and classified the features. A final GUI was then wire-framed which was based on the paper prototype

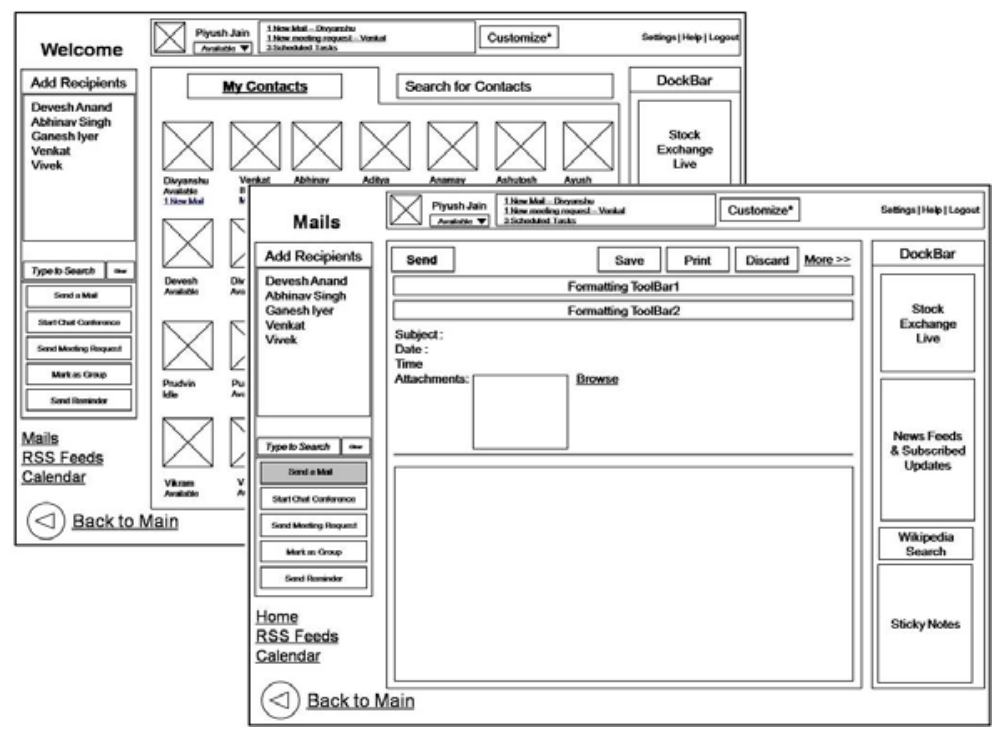

Fig. 3. Wireframes for various screenshots

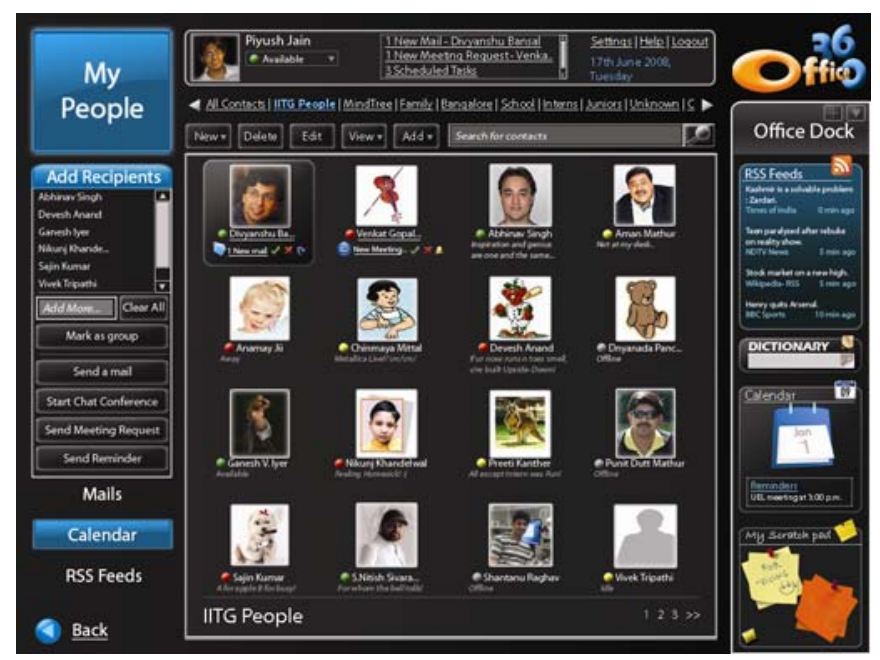

Fig. 4. A screen shot of the final GUI 
that stood closest to our findings. Static and non functional dynamic prototypes of the screenshots were made (Fig 3). A screen shot simulation of the final GUI is shown in Fig 4. 'Contacts' are shown as large thumbnails with images. A task bar at the top shows new updates, settings etc. In the proposed design, Contacts can be searched dynamically and added to a recipient box where the user can then select the mode of communication. The DockBar shows the different docklets as per the user's preferences Contacts are selected as recipients and the action 'Send a Mail' is selected. Attachment's previewing can be noticed. The interface uses lesser buttons and actions up fronted on the screen thereby reducing its clumsiness in general.

\section{Conclusions}

We had derived from the user survey the attributes and the need for Tailorability of the new system and the activity flow of performing a task without having to go out of the hierarchical level in the information architecture. Multiplicity of office products with communicating features embedded resulted in unsatisfactory situation for users. What they preferred, from the point of view of usability, is an independent dock-able plugin type solution that could be used regardless of the software they were currently using on the screen and could be customized as per their own specific contextual requirements

Acknowledgments. We would like to acknowledge MindTree Ltd., Bangalore and its participating employees for providing the test cases for our research. We extend our special acknowledgements to Mr. Sannidhya Misra and the entire User Experience Group of MindTree Ltd. for providing their valuable feedbacks and guidance throughout our research.

\section{References}

1. Uden, L., Kumaresan, A.: Usable Collaborative Email Requirements Using Activity Theory. Informatica 31, 71-83 (2006/2007)

2. Jennings III, R.B., Nahum, E.M., Olshefski, D.P., Saha, D., Shae, Z.-Y.: A Study of Internet Instant Messaging and Chat Protocols. IEEE Network 20(4), 16-21 (2006)

3. John, B.E., Bass, L., Sanchez-Segura, M.-I., Adams, R.J.: Bringing Usability Concerns to the Design of Software Architecture. In: Proceedings of Engineering Human Computer Interaction and Interactive Systems Joint Working Conference - EHCI-DSVIS 2004, Hamburg, Germany, July 11-13 (2004)

4. Boyd, d.m., Ellison, N.B.: Social network sites: Definition, history, and scholarship. Journal of Computer Mediated Communications 13(1), article 11 (2007)

5. Fox, D., Naidu, S.: Usability Evaluation of Three Social Networking Sites. Usability News 11(1) (April 2009)

6. Dix, A., Cowen, L.: HCI 2.0? Usability meets Web 2.0. In: Proceedings of HCI 2007, vol. 2. British Computer Society (2007)

7. Nakayama, T.: An Office Communications Model using CTstage4i. OKI Technical Review 70(1) (193) (January 2003) 
8. Parkulo, M.A., Mentel, J.J., Murray, L., Willard, C.H.M.: An Intra Office Email System in a Primary Care Practice: Implementation Challenges and Solutions. In: AMIA 2001; Annual Symposium Proceedings - Vision of the Future and Lessons of the Past, Washington DC (2001)

9. Avison, D.E., Wood-Harper, T.: Multiview: An Exploration in Information System Development. Oxford University Press, Oxford (2008)

10. The E-mail Management Crisis: New Research on the Seven Critical E-mail Management Problems, IT White Paper News Letter, Dell Message One (March 2009)

11. Email Archiving \& Compliance Solutions for mid-sized organizations, IT White paper published by IBM-America: June 15 (2009), http: / / www . ibm. com

12. Bass, L., John, B.E., Kates, J.: Achieving Usability Through Software Architecture. In: Proceedings of EHCI -DSVIS 2004 - 9th IFIP Working Conference on Engineering for Human Computer Interaction, July 11-13 (2004) 